\title{
Short-Wavelength Blue Light Contributes to Pyroptosis of Human Lens Epithelial Cells (hLECs) by Caspase-1-GSDMD Signalling Axis Activation
}

\section{Zhaowei Song}

First Affiliated Hospital of Harbin Medical University

\section{Xiaohui Wang}

First Affiliated Hospital of Harbin Medical University

Huazhang Li

First Affiliated Hospital of Harbin Medical University

\section{Ying Sun}

First Affiliated Hospital of Harbin Medical University

Kexin Liu

First Affiliated Hospital of Harbin Medical University

Xiangyu Liu

First Affiliated Hospital of Harbin Medical University

\section{Sheng Su}

First Affiliated Hospital of Harbin Medical University

Zhijian Li ( Izj6515@sina.com )

First Affiliated Hospital of Harbin Medical University

\section{Research}

Keywords: Pyroptosis, Short-wavelength blue light, Caspase-1, GSDMD, Cataract

Posted Date: July 23rd, 2020

DOI: https://doi.org/10.21203/rs.3.rs-44771/v1

License: (1) (1) This work is licensed under a Creative Commons Attribution 4.0 International License. Read Full License 


\section{Abstract}

Backgroud: To examine the effects of short-wavelength blue light (SWBL) on cultured human lens epithelial cells (hLECs). The nosogenesis of cataracts after SWBL exposure was discussed.

Methods: HLE-B3 hLECs were divided into 3 groups randomly: A: normal control group, which consisted of hLECs cultured in the dark; B: the caspase-1 inhibitor group; and C: the SWBL exposure group. After the SWBL (2500 lux) irradiation (for 8, 16, 24, and $32 \mathrm{~h}$ ), the caspase-1 and gasdermin D (GSDMD) expression levels in HLE-B3 hLECs were examined using ELISA, immunofluorescence, and Western blotting analyses. Double-positive staining of HLE-B3 hLECs for activated and inhibited caspase-1 was used to confirm pyroptosis in hLECs by flow cytometry.

Results: SWBL can cause cell death in HLE-B3 hLECs, but a caspase-1 inhibitor suppressed cell death. The flow cytometry results also confirmed the does-dependent of short-wavelength blue light irradiation on pyroptotic death of hLECs. Caspase-1 and GSDMD expression levels of all hLECs groups changed with short-wavelength blue light exposure times $(8,16,24$, and $32 \mathrm{~h}$ ) and were higher in groups $B$ and $C$ than group $A$. The immunofluorescence results demonstrated that the expression of GSDMD-N was higher in the cell membrane in both the $\mathrm{B}$ and $\mathrm{C}$ groups than in the $\mathrm{A}$ group.

Conclusion: The data indicate that SWBL induces pyroptotic programmed cell death by activation of the GSDMD signalling axis in HLE-B3 hLECs. These results provide new insights into the exploitation of new candidates for the prevention of cataracts.

\section{Background}

Cataract remains the leading cause of treatable blindness in the world, are characterized by opacification of the lens, and account for nearly half of all blindness cases[1]. Currently, cataract extraction and intraocular lens (IOL) implantation are the main treatment methods for cataracts. However, surgery of cataract might bring many serious postoperative complications, such as infection, corneal oedema, and high intraocular pressure. Poor postoperative outcomes, cost, and fear of cataract surgery are the most common barriers to cataract surgery $[2,3]$. Therefore, it is necessary to elucidate pathogenic mechanisms of cataracts from a new point of view and further provide new targets for effective prevention strategies.

To date, pathogenesis of cataract is not clearly elucidated. A previous study showed that endogenous photosensitive substances in the lens, such as tryptophan and riboflavin, are extremely sensitive to shortwavelength light, which includes UVA and short-wavelength blue light [4].

It is estimated that artificial light sources, including fluorescent light tubes and LEDs, consumed approximately $19 \%$ of the electricity produced worldwide in 2012[5]. SWBL mainly comes from artificial light sources. With the widespread use of blue backlight electronic display devices, such as computers and mobile, the human eyes are increasingly exposed to more SWBL[6]. 
SWBL (400-500 nm), induced oxidative stress and cellular injury, has attracted increasing attention because of the probably injury to the retina [7-9]. The transmission of visible light, especially in the blue spectrum region, is significantly reduced with age. Majority of them is absorbed in lens $[10,11]$. It has been suggested a link between exposure to SWBL and the development of cataracts, but additional studies are needed to confirm it.

According to the classification established by the NCCD, cell death is roughly classified as apoptosis, autophagic cell death, necrosis, pyroptosis, etc [12]. In the pathogenesis of cataracts, at least three types of programmed cell death pathways have been studied in deed, including apoptosis[13], autophagy[14], and pyroptosis[15]. The formation of cataracts is not entirely dependent on any single programmed cell death pathway. Of all the types of programmed cell death, apoptosis is the most well understood forms of death. Apoptosis of LECs is a common cellular basis for the formation of cataracts[15]. However, the specific pyroptosis pathway implicated in cataract formation remains to be elucidated.

Pyroptosis and apoptosis depend on particular caspases to motivate their separate programmed cell death pathways. Pyroptosis can be caused by both canonical and noncanonical inflammasome signalling pathways [15-21]. Caspase-1, which leads to the cleavage of GSDMD and induces pore opening on the cytomembrane[22-24], can activate canonical inflammasome signalling pathway.

Our previous animal study indicated that pyroptosis plays a vital role in cataracts formation caused by SWBL exposure. In the present study, SWBL exposure systems were established to discuss the photobiological effects on cultured human LECs, including morphological changes, cell viability and protein levels, as well as the localization of caspase-1 and GSDMD. The results indicated that the caspase-1/GSDMD signalling axis may be involved in the pathological process under SWBL exposure.

\section{Methods}

\section{Cell culture}

The HLE-B3 hLEC line (ATCC, Manassas, VA, USA) was cultured in DMEM containing $10 \% \mathrm{FBS}$ at $37^{\circ} \mathrm{C}$. Under these conditions, the doubling time of the cells was about 24 hours and the cells passage rate were generally $1: 2$. The cells were inoculated onto 6 -well plates $\left(5 \times 10^{5}-1 \times 10^{6}\right.$ cells $)$ for the experiments.

We used AC-YVAD-CMK as the caspase-1 inhibitor and evaluated its protective effects against shortwavelength blue light exposure. The concentration of AC-YVAD-CMK was decided by the cell counting kit8 assay (SAB, USA)[25]. Detailed methods are provided in the supplementary materials.

HLE-B3 LECs were divided into 3 groups randomly: A: normal control group, which consisted of HLE-B3 cells cultured in the dark; $\mathrm{B}$ : the caspase-1 inhibitor group; and C: the short-wavelength blue light exposure. Groups A, B, and C were subdivided into four groups according to blue light exposure times (8, $16,24$, and $32 \mathrm{~h})$. In the caspase-1 inhibitor group, hLECs were treated with ACYVADCMK $(20 \mu \mathrm{mol} / \mathrm{L})$. 


\section{Short-wavelength blue light exposure}

An illuminance intensity of 2500 lux was used to study the potential influence of SWBL on HLE-B3 hLECs in vitro [26]. Detailed methods are provided in the supplementary materials.

\section{Cell morphology analysis}

The number and dynamic morphological changes of HLE-B3 hLECs were observed under an inverted microscope $(\times 200)$. HLE-B3 hLECs morphological changes in both groups were photographed and compared after $8,16,24$ and $32 \mathrm{~h}$.

\section{Enzyme-linked immunosorbent assay}

The protein expressions of caspase-1, -4 , and GSDMD in the three groups were decided by using individual ELISA kits. Detailed methods are provided in the supplementary materials.

\section{Flow cytometry}

To distinguish pyroptosis from apoptosis in HLE-B3 hLECs, a cell viability assay was performed by flow cytometry [28] The assay was repeated 3 times. Detailed methods are provided in the supplementary materials.

\section{Immunofluorescence localization}

Immunohistochemistry was performed to measure the expression levels of caspase- 1 and GSDMD. The fluorescence images were further processed using Image-Pro plus 6.0. Six random sections per smear at a magnification of $\times 400$ over a microscopic field were observed within $1 \mathrm{~h}$ [29]. Detailed methods are provided in the supplementary materials.

\section{Western blot analysis}

Western blot was applied to determine protein expression levels that were previously described by Mei, et al [30]. Band intensities were analysed with ImageJ software (NIH, Bethesda, MD, USA). Detailed methods are provided in the supplementary materials.

\section{Statistical analyses}


SPSS 23.0 statistical software (SPSS Inc., USA) was used to perform statistical analysis. The results are presented as the mean \pm SD. Data were analysed by two-way ANOVA. A value of $p<0.05$ was considered statistically significant.

\section{Results}

\section{Short-wavelength blue light can cause cell death, but ACYVADCMK suppresses HLE-B3 hLECs death}

The number and dynamic morphological changes in HLE-B3 hLECs were observed. After $8 \mathrm{~h}$ of SWBL exposure, the number of cells in group B was significantly decreased, and there were no prominent morphological changes. However, the number of HLE-B3 hLECs in group $\mathrm{C}$ decreased by a moderate amount, and the cells were slightly swollen. After $24 \mathrm{~h}$ of exposure, the number of hLECs decreased significantly, and the cells were obviously swollen in group C. After $32 \mathrm{~h}$ of exposure, the number of hLECs in groups B and C was significantly decreased. Furthermore, the HLE-B3 hLECs in group B were swollen, and the LECs in group $\mathrm{C}$ were severely swollen and morphologically deformed (Fig. 1).

Double-positive staining for activated and inhibited caspase-1 using flow cytometry was selected to assess pyroptotic programmed cell death. As shown in Fig. 2, in the B group, the proportions of doublepositive hLECs were increased at $8,16,24$, and $32 \mathrm{~h}$ compared with those in the control group. Although there was no significant difference at $16 \mathrm{~h}$ compared to $8 \mathrm{~h}$, the proportion of double-positive hLECs was increased at a does-dependent manner. In the short-wavelength blue light group, the proportion of doublepositive hLECs was also significantly increased at $8,16,24$, and $32 \mathrm{~h}$ in a does-dependent manner $(\mathrm{p}<$ 0.05). Moreover, the proportions of double-positive hLECs were increased at $8,16,24$, and $32 \mathrm{~h}$ in the short-wavelength blue light exposure subgroups compared with the same treatment times in the ACYVADCMK subgroups $(p<0.05)$. Our flow cytometry results also confirmed that $S W B L$ exposure motivated pyroptotic death in HLE-B3 hLECs at a does-dependent manner and that ACYVADCMK suppressed cell death.

\section{Short-wavelength blue light initiates caspase-1-induced pyroptosis of hLECs in culture}

Enzyme-linked immunosorbent assay (ELISA) was performed and revealed that the expression levels of caspase- 1 and GSDMD in all LEC groups changed with the short-wavelength blue light exposure time (8, 16,24 , and 32 h); caspase- 1 and GSDMD expression levels were significantly increased in ACYVADCMK groups and SWBL group compared with control group. (Fig. 3a, b). Interestingly, we found that the expression of caspase-4, a member of a cysteine protease family in humans, was also significantly suppressed when the caspase-1 inhibitor was used (Fig. 3c). 
Next, we performed immunohistochemical assays to examine the expression levels of caspase-1 and GSDMD-N. The data showed that SWBL exposure activated the expression of both proteins. The protein expressions of caspase-1 p20 and GSDMD in ACYVADCMK and SWBL group were increased. However, ACYVADCMK significantly reversed the increase in expression compared to that of the SWBL group (Fig. 4).

Western blot was performed to examine the influence of ACYVADCMK on caspase-1 and GSDMD-N protein expression (Fig. 5). The protein expression levels (Fig. 5) of caspase-1 and GSDMD-N in the SWBL exposure group were increased compared to those in the control group $(p<0.05)$. The inhibitor-treated group exhibited reversal of the increased expression levels of caspase- 1 compared to that of the SWBL exposure group. The results suggest that SWBL exposure could activate caspase- 1 and GSDMD in cultured HLE-B3 hLECs. However, AC-YVAD-CMK can reduce caspase-1-induced pyroptosis of hLECs in culture and suppressing the progression of cataracts.

\section{Short-wavelength blue light activates GSDMD-N in the HLE- B3 cell membrane via caspase-1}

The immunofluorescence results showed that in the control group, both caspase-1 and GSDMD-N proteins were expressed in the cytoplasm rather than in other organelles. Compared to that of the group A, the protein expression levels of caspase-1 in the cytoplasm increased with increasing exposure to SWBL, and the distribution was not uniform, but the caspase-1 protein was not expressed on the cell membrane or in the nucleus. However, the protein expression of GSDMD-N was increased in the cell membrane in both the short-wavelength blue light and caspase-1 inhibitor groups (Fig. 6).

\section{Discussion}

To reduce the occurrence of sightlessness and delay the onset of cataracts, it is vital to determine cataract pathogenesis. In recent decades, researches have focused on the pyroptosis signalling pathways in a varity of diseases, which is implicated in this process. Recently, an in vitro study of LECs indicated that the caspase- $1 / \mathrm{IL}-1 \beta$ signalling pathways may be involved in the pathological process of cataract formation [15]. Data presented in a previous report demonstrated that pyroptosis plays a vital role in cataracts formation under SWBL exposure in vivo. In this study, we further investigated the influences of caspase-1/GSDMD on HLE-B3 hLECs in culture and the underlying pathogenesis of cataract formation under SWBL exposure. The results of this study showed that SWBL could initiate caspase-1-induced pyroptosis of hLECs. LEC dysfunction may lead to oedema in superficial cortical lens fibres, which in turn may lead to mature cataracts [31].

Caspase-1, a representative marker in the pyroptosis signalling pathway, is an inflammatory caspase that is activated in not only immune cells but also mesenchymal and epithelial cells [32]. The most important function of cleaved caspase- 1 is to facilitate GSDMD cleavage into an N-terminal effector domain and a 
C-terminal inhibitory domain. The N-terminal domain can bind to PIPs in the cell membrane and promote the release of intracellular inflammatory substances, such as IL-1 $\beta$, through pore opening in cell membrane, ultimately leading to pyroptosis [33]. In agreement with the previous reports, this research results confirmed that caspase- 1 and GSDMD protein expression levels, determined by ELISA, immunofluorescence and western blot, were increased in HLE-B3 hLECs at a does-dependent manner, as. As expected, AC-YVAD-CMK effectively reversed the increased expression trend of GSDMD compared to that of the SWBL exposure group. Our study identified for the first time that SWBL could induce caspase1-mediated pyroptosis in HLE-B3 LECs in vitro. This work further suggests that AC-YVAD-CMK may be an effective reagent to suppress the formation of cataracts and defend against LEC damage by suppressing the caspase-1/GSDMD pathways in response to SWBL exposure. Our results demonstrated that the higher expression of human caspase-4, which has the highest homology to murine caspase-11[22], was also significantly suppressed when a caspase- 1 inhibitor was used. Theoretically, murine caspase- 11 and its human orthologue caspase- 4 are activated in noncanonical pyroptosis. Caspase- 1 knockout mice lack both caspase-11 and caspase-1 [34, 35]. In this study, the caspase-1 inhibitor may also have suppressed the expression of caspase- 4 due to the close genomic location of caspase- 1 and caspase- 4 . Further studies are needed to investigate their relative effects in response to SWBL exposure.

Although the increased expression of caspase-1 and GSDMD and the inhibitory activity of caspase-1 indicate that pyroptosis may be involved in the cataract formation under SWBL exposure, pyroptosis and apoptosis are still difficult to distinguish. It has been reported that apoptosis is involved in cataract formation ${ }^{18}$. Multiple studies show that the characteristic morphological changes of various types of cell death are cell rounding and shrinkage in apoptosis, intact cell membrane and vacuolated cytoplasm in autophagy, and the appearance of membrane bubbles or swelling in pyroptosis [36, 37].

The number and types of morphological changes in HLE-B3 hLECs were observed under an inverted microscope in our study. During the 32-hour observation period, there were no significant changes in the number and status of cells in the control group. However, after $32 \mathrm{~h}$ of exposure, the numbers of LECs in the AC-YVAD-CMK group and SWBL exposure group were significantly decreased, and the LECs were swollen in the AC-YVAD-CMK group and severely swollen and deformed morphologically in the blue light exposure group. Flow cytometry, a strength of this work, was used to assess pyroptotic programme cell death based on double-positive staining for activated and inhibited caspase-1. Flow cytometry were selected to analyse the ratio of viable cells and the degree of pyroptosis of HLE-B3 cells after exposure to SWBL for different times. Our results suggested that SWBL exposure causes pyroptotic death in HLE-B3 hLECs in a does-dependent manner.

Some potential limitations of the present study need to be addressed, however. First, the conclusion has some limitations because of the relatively lower knockout efficiency of caspase-1 by AC-YVAD-CMK and further studies are required. Second, although the morphological changes, double-positive staining to indicate pyroptotic cell death, protein levels, and localization of caspase-1 and GSDMD were elucidated in our study, these results, which do not include mRNA data, seem to be insufficient to reveal the exact molecular mechanisms. Third, direct evidence of plasma membrane rupture when pyroptosis occurs was 
not examined. Additional studies, such as transmission electron microscopy observations of the cell membrane, are needed.

\section{Conclusion}

Our study showed that short-wavelength blue light induces pyroptotic programmed cell death by activation of the caspase-1/GSDMD signalling axis in HLE-B3 hLECs. These findings provide evidence and novel insights for new drug candidates for the prevention and treatment of cataracts.

\section{Abbreviations}

LECs: lens epithelial cells; SWBL: short-wavelength blue light; hLECs: human lens epithelial cells; GSDMD: gasdermin D; LED: light-emitting diode; IOLs: intraocular lenses; NCCD: Nomenclature Committee on Cell Death; PBS: phosphate-buffered saline; ATCC: American Type Culture Collection; CCK-8: cell counting kit8; LPS: lipopolysaccharide; GAPDH: glyceraldehyde 3-phosphate dehydrogenase; NIH: National Institute of Health, and PI: propidium iodide.

\section{Declarations}

\section{Ethics approval and consent to participate}

All experiment protocols were approved by the First Affiliated Hospital of Harbin Medical University, Harbin, China.

\section{Consent for publication}

Not available.

\section{Availability of data and materials}

The datasets used during this current study are available from the corresponding author on reasonable request.

\section{Competing interests}

The authors declare that they have no competing interests.

\section{Funding}


This study was supported by the National Natural Science Foundation of China (Grant No. 81870643 and 81800811). The funding bodies participated in the design of the study, analysis and interpretation of the data.

\section{Authors' contributions}

$\mathrm{ZS}, \mathrm{XW}, \mathrm{ZL}$, and $\mathrm{YS}$ performed the in vitro experiments. $\mathrm{KL}, \mathrm{XL}$, and $\mathrm{SS}$ analyzed the data. $\mathrm{ZL}$ designed the research and wrote the manuscript. All authors reviewed and commented on the manuscript. All authors read and approved the final manuscript.

\section{Acknowledgments}

Not Applicable

\section{References}

1. Flaxman SR, Bourne RA, Resnikoff S, Ackland P, Braithwaite T, Cicinelli MV, et al. Global causes of blindness and distance vision impairment 1990-2020: a systematic review and meta-analysis. Lancet Glob Health.2017;5:e1221-e1234.

2. Meacock WR, Spalton DJ, Boyce J, \& Marshall J. The effect of posterior capsule opacification on visual function. Invest Ophthalmol Vis Sci. 2003; 44:4665-4669.

3. Li Z, Song Z, Wu S, Xu K, Jin D, Wang H, et al. Outcomes and barriers to uptake of cataract surgery in rural northern China: the Heilongjiang Eye Study. Ophthalmic Epidemiol. 2014;21:161-8.

4. Yang MY, Chang CJ, Chen LY. Blue light induced reactive oxygen species from flavin mononucleotide and flavin adenine dinucleotide on lethality of HeLa cells. Photochem Photobiol B. 2017;173:325332.

5. Behar-Cohen, F., Martinsons C, Viénot F, Zissis G, Barlier-Salsi A, Cesarini JP, et al. Light-emitting diodes (LED) for domestic lighting: Any risks for the eye? Prog Retin Eye Res. 2011;30:239-257.

6. Leung TW, Li RW, Kee CS. Blue-Light Filtering Spectacle Lenses: Optical and Clinical Performances. PLoS One. 2017;12:e0169114.

7. Emi Ooe, Yoshiki Kuse, Tomohiro Yako, Tetsuya Sogon, Shinsuke Nakamura, Hideaki Hara, et al. Bilberry extract and anthocyanins suppress unfolded protein response induced by exposure to blue LED light of cells in photoreceptor cell line. Mol Vis. 2018; 24:621-632.

8. Song JA, Choi CY. Effects of blue light spectra on retinal stress and damage in goldfish (Carassius auratus). Fish Physiol Biochem. 2019;45:391-400.

9. Kernt M, Walch A, Neubauer AS, Hirneiss C, Haritoglou C, Ulbig MW, et al. Filtering blue light reduces light-induced oxidative stress, senescence and accumulation of extra-cellular matrix proteins in human retinal pigment epithelium cells. Clin Experiment Ophthalmol. 2012;40: e87-e97. 
10. van de Kraats J, van Norren D. Optical density of the aging human ocular media in the visible and the UV. J Opt Soc Am A Opt Image Sci Vis. 2007;24 (7): 1842-1857.

11. Kessel L, Lundeman JH, Herbst K, Andersen TV, Larsen M. Age-related changes in the transmission properties of the human lens and their relevance to circadian entrainment. J Cataract Refract Surg. 2010;36:308-312.

12. Galluzzi L, Vitale I, Abrams JM, Alnemri ES, Baehrecke EH, Blagosklonny MV, et al. Molecular definitions of cell death subroutines: recommendations of the Nomenclature Committee on Cell Death 2012. Cell Death Differ. 2012;19: 107-120.

13. Gong W, Zhu G, Li J, Yang X. LncRNA MALAT1 promotes the apoptosis and oxidative stress of human lens epithelial cells via p38MAPK pathway in diabetic cataract. Diabetes Res Clin Pract. 2018:144:314-321.

14. Li D, Liu GQ, Lu PR. High glucose: activating autophagy and affecting the biological behavior of human lens epithelial cells. Int J Ophthalmol. 2019;12(7): 1061-1066.

15. Jin X, Jin H, Shi Y, Guo Y, Zhang H. Pyroptosis, a novel mechanism implicated in cataracts. Mol Med Rep. 2018;18(2):2277-2285.

16. Godley BF, Shamsi FA, Liang FQ, Jarrett SG, Davies S, Boulton M. Blue light induces mitochondrial DNA damage and free radical production in epithelial cells. Journal of Biological Chemistry. 2005;280:21061-21066.

17. S. Beatty, H.-H. Koh, M. Phil, D. Henson. \& M. Boulton. The role of oxidative stress in the pathogenesis of age-related macular degeneration. Survey of Ophthalmology. 2000;45: 115-134.

18. Bai J, Yang F, Dong L, Zheng Y. Ghrelin Protects Human Lens Epithelial Cells against Oxidative Stress-Induced Damage. Oxid Med Cell Longev. 2017;2017:1910450.

19. He Y, Hara H, Nune G. Mechanism and regulation of NLRP3 inflammasome activation. Trends Biochem Sci. 2016; 41:1012-1021.

20. Jiang D, Chen S, Sun R, Zhang X, Wang D. The NLRP3 inflammasome: role in metabolic disorders and regulation by metabolic pathways. Cancer Lett. 2018;419:8-19.

21. C Dempsey, A Rubio Araiz, K J Bryson, O Finucane, C Larkin, EL Mills, et al. Inhibiting the NLRP3 inflammasome with MCC950 promotes non-phlogistic clearance of amyloid-beta and cognitive function in APP/PS1 mice. Brain Behav Immun. 2017;61:306-316.

22. Shi J, Zhao Y, Wang Y, Gao W, Ding J, Li P, et al. Inflammatory caspases are innate immune receptors for intracellular LPS. Nature. $2014: 514$ :187-192.

23. Uchiyama R, Tsutsui $H$. Caspases as the key effectors of inflammatory responses against bacterial infection. Arch Immunol Ther Exp. 2015;63:1-13.

24. S. L. Fink and B. T. Cookson. Caspase-1-dependent pore formation during pyroptosis leads to osmotic lysis of infected host macrophages. Cellular Microbiology. 2006; 8:1812-1825.

25. Wang J, Fang Y, Liu T. TRIM32 Promotes the Growth of Gastric Cancer Cells through Enhancing AKT Activity and Glucose Transportation. Biomed Res Int. 2020;2020:4027627. 
26. Xie C, Li X, Tong J,Gu Y and Shen Y. Effects of white light-emitting diode (LED) light exposure with different Correlated Color Temperatures (CCTs) on human lens epithelial cells in culture. Photochemistry and Photobiology. 2014;90: 853-859.

27. Cheng T, Xu M, Qin B, Wu J, Tu Y, Kang L, et al. IncRNA H19 contributes to oxidative damage repair in the early age-related cataract by regulating miR-29a/TDG axis. Cell Mol Med. 2019;23:6131-6139.

28. Kong DL, Kong FY, Liu XY, Yan C, Cui J,Tang RX, et al. Soluble egg antigen of Schistosoma japonicum induces pyroptosis in hepatic stellate cells by modulating ROS production. Parasit Vectors. 2019;12:475.

29. Singh GB, Kshirasagar N, Patibandla S, Puchchakayala G, Koka S, and Boini KM, et al. Nicotine instigates prodocyte injury via NLRP3 inflammasomes activation. Aging (Albany NY). 2019;11:12810-12821.

30. Mei Y,Tang Z,Li Z, Yang X. Repeatability and Reproducibility of Quantitative Corneal Shape Analysis after Orthokeratology Treatment Using Image-Pro Plus Software. J Ophthalmol. 2016;2016: 1732476.

31. Inanc M, Tekin K, Erol YO, Sargon MF, Koc M, Budakoglu O, et al. The ultrastructural alterations in the lens capsule and epithelium in eyes with traumatic white cataract. Int Ophthalmol. 2019:39:47-53.

32. Yazdi AS, Drexler SK and Tschopp J. The role of the inflammasome in nonmyeloid cells. J Clin Immunol. 2010;30: 623-627.

33. Yang M, Yang X, Wang S, Xu L, Ke S, Ding X, et al. HMGB1- induced Endothelial cell pyroptosis is involved in Systemic inflammatory responsesyndrome following radiofrequency ablation of hepatic hemangiomas. Am J Trans/ Res. 2019;11:7555-7567.

34. Man SM, Karki R, Briard B, Burton A, Gingras S, Pelletier S, et al. Differential roles of caspase-1 and caspase-11 in infection and inflammation. Sci Rep. 2017;7:45126.

35. Kayagaki N, Warming S, Lamkanfi M, Walle LV, Louie S, Dong J, et al. Non-canonical inflammasome activation targets caspase-11. Nature. 2011; 479;117-121.

36. Fink S.L., Cookson B.T. Apoptosis, pyroptosis, and necrosis: Mechanistic description of dead and dying eukaryotic cells. Infect. Immun. 2005;73:1907-1916.

37. Teng JF, Mei QB, Zhou XG, Tang Y, Xiong R, Qiu WQ, et al. Polyphyllin VI Induces Caspase-1Mediated Pyroptosis via the Induction of ROS/NF-KB/NLRP3/GSDMD Signal Axis in Non-Small Cell Lung Cancer. Cancers (Basel).2020 Jan; 12(1): 193

\section{Figures}




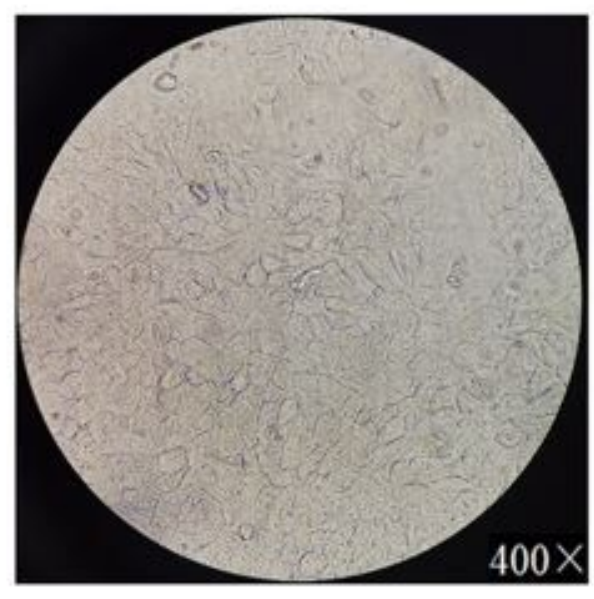

A

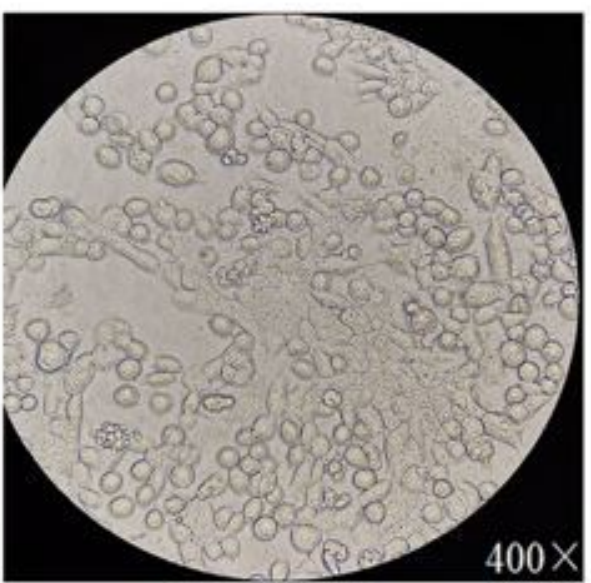

B

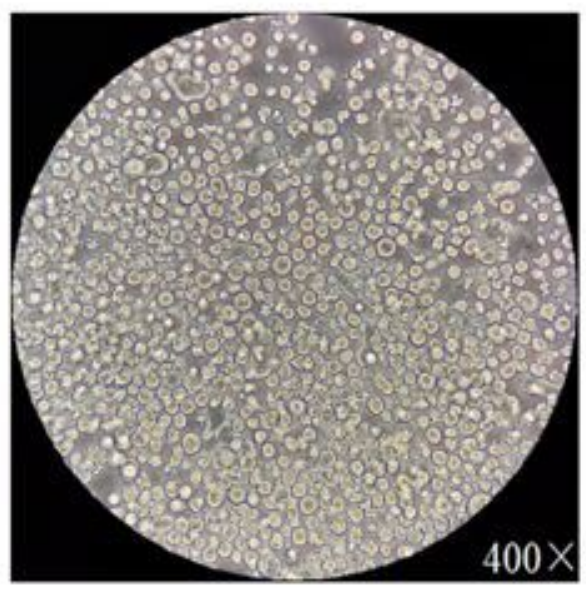

C

\section{Figure 1}

Representative images of LECs at $32 \mathrm{~h}$ under an inverted microscope $(\times 200)(a)$ no significant change in the cells in the control group. (b) The cell number decreased significantly, and the cells were swollen in the AC-YVAD-CMK treatment group. (c) The cell number decreased significantly, and the cells were severely swollen and deformed morphologically. 
a
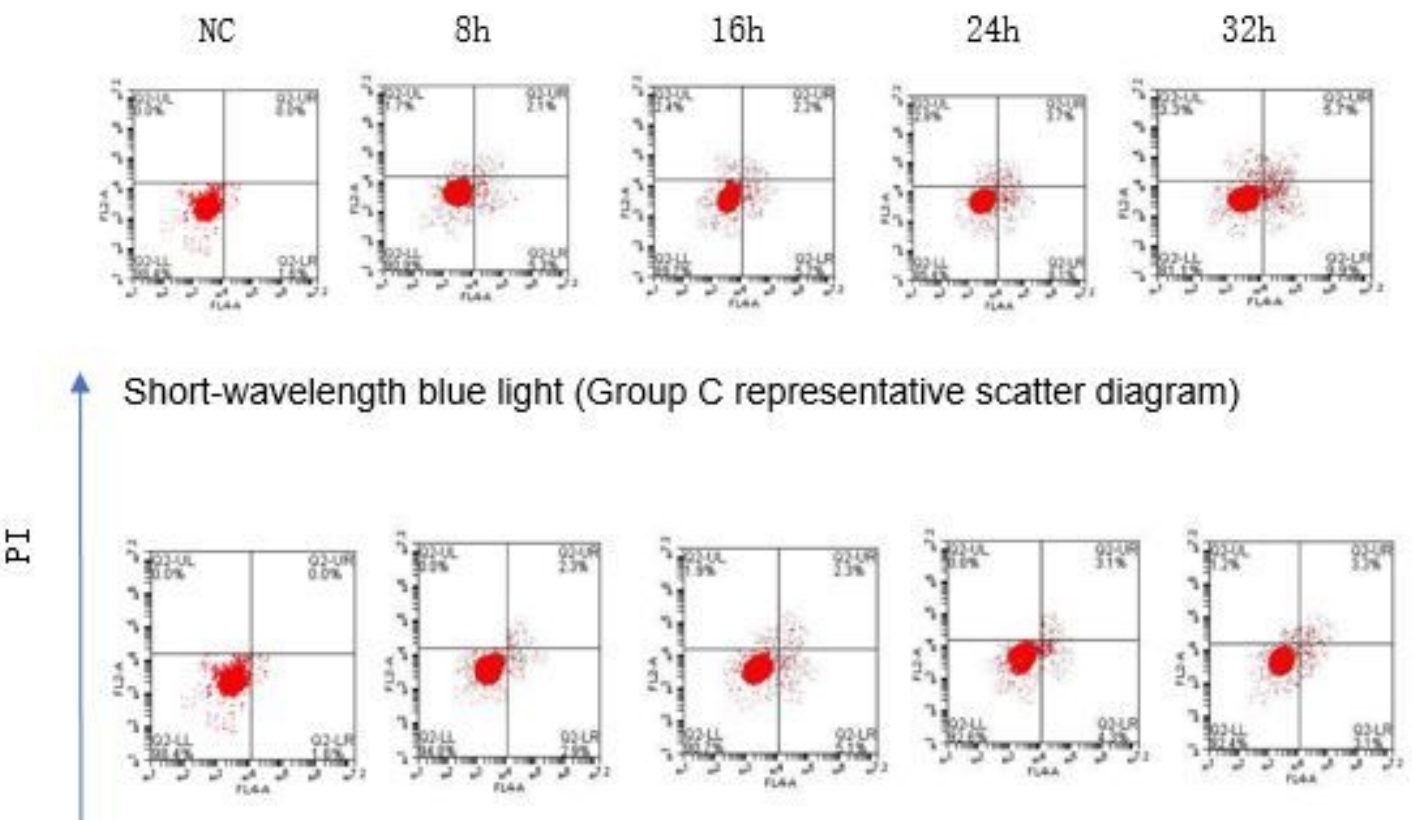

Caspase-1 inhibitor (Group B representative scatter diagrama Anncxia $V$

\section{b}
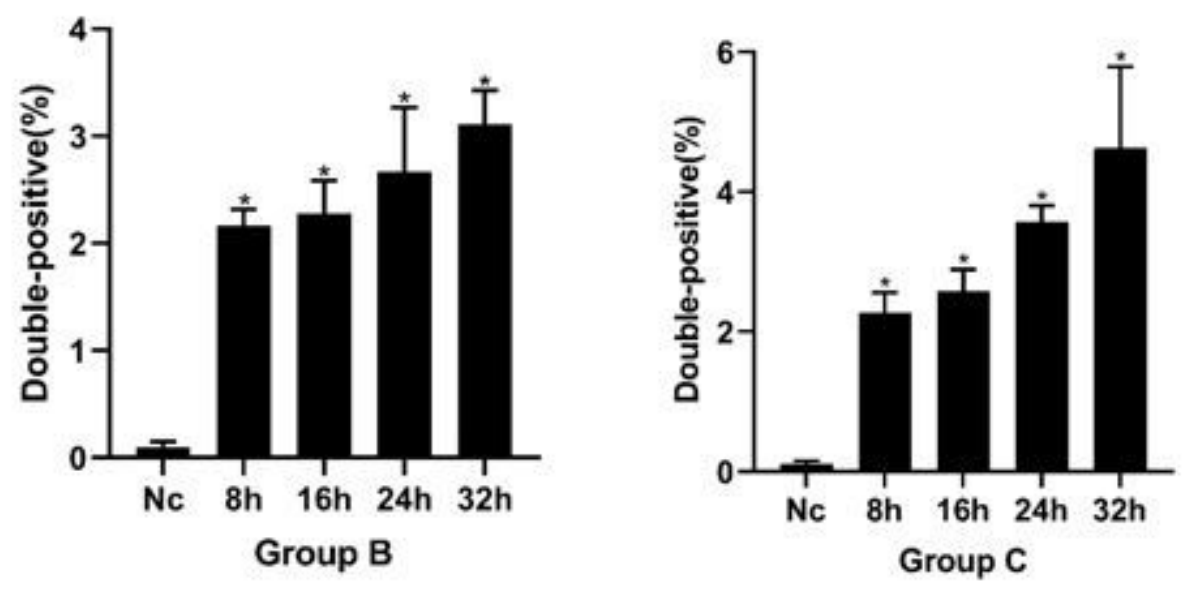

Figure 2

Double-positive staining of inhibited and activated caspase- 1 and flow cytometry analysis. (a) Representative images of double-positive staining were detected by flow cytometry. (b) The doublepositive cells proportions. Group B: caspase-1 inhibitor group; Group C: short-wavelength blue light group; $\mathrm{NC}$, normal control group; the proportion of double-positive hLECs was increased at 8, 16, 24 and $32 \mathrm{~h}$ in a dose-dependent manner in both groups B and C. $n=5$. * vs. NC $p<0.05$. 
a

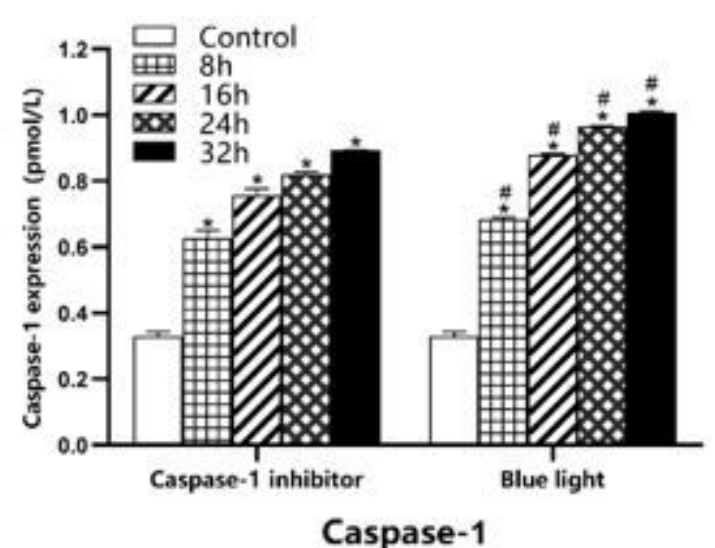

C

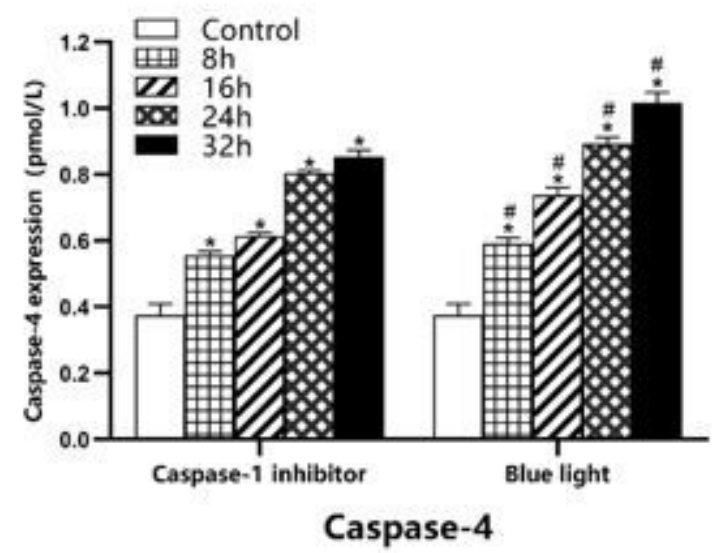

b

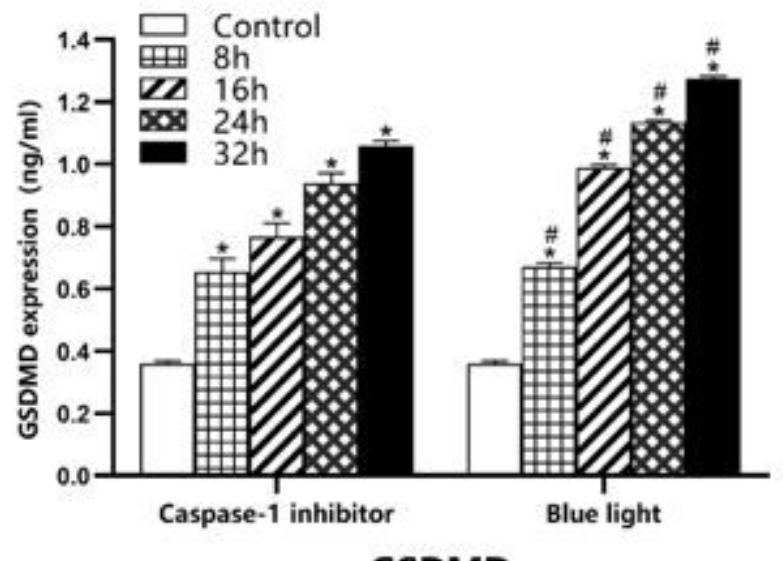

GSDMD

\section{Figure 3}

The levels of caspase-1, GSDMD, and caspase- 4 in HLE-B3 cells at different times were determined using ELISA. (a) The level of caspase-1 in HLE-B3 hLECs of the AC-YVAD-CMK group and short-wavelength exposure group. (b) The level of GSDMD in HLE-B3 cells in the AC-YVAD-CMK group and shortwavelength exposure group. (c) The level of caspase-4 in HLE-B3 cells in the AC-YVAD-CMK group and short-wavelength exposure group. Compared with the levels in the control group, the caspase-1, GSDMD, and caspase-4 levels in both the caspase- 1 inhibitor and short-wavelength groups increased. $n=5$. * vs. the control group, $p<0.05$. \# vs. the same time point in the AC-YVAD-CMK group, $p<0.05$. 
a
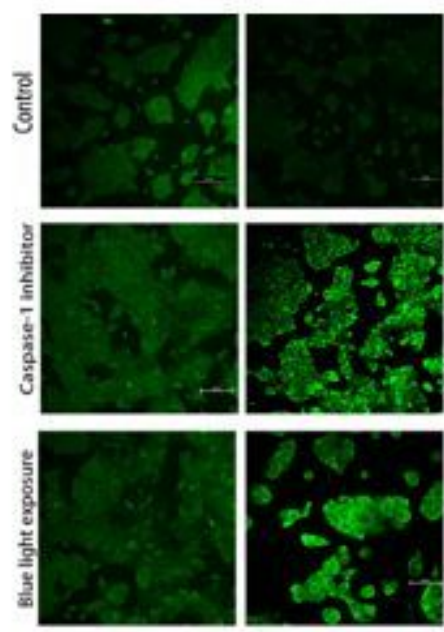

$8 \mathrm{~h}$

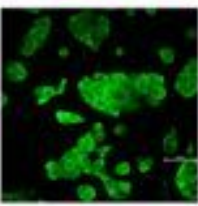

$16 \mathrm{~h}$

C
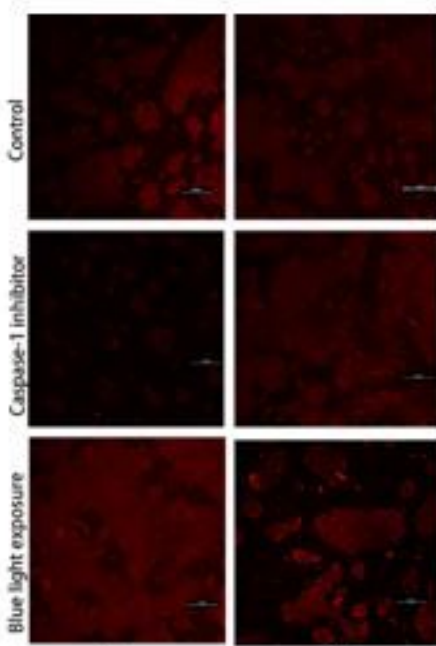

$8 h$

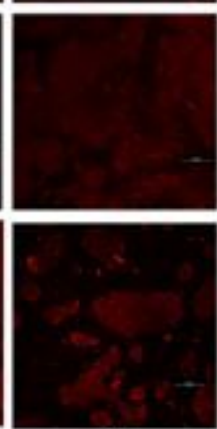

$16 \mathrm{~h}$
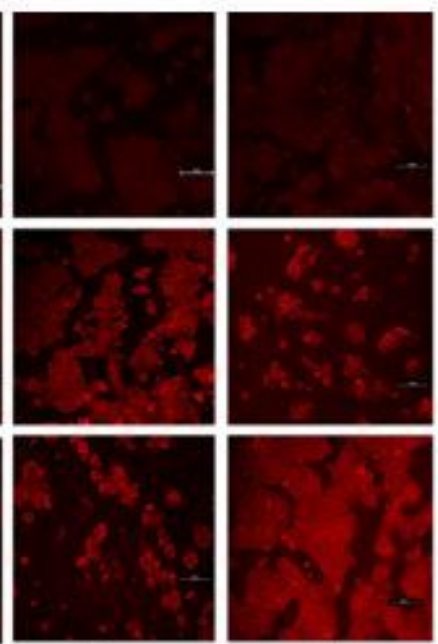

$24 h$
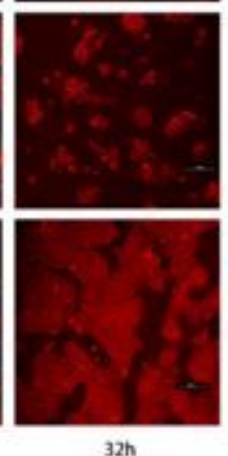

b

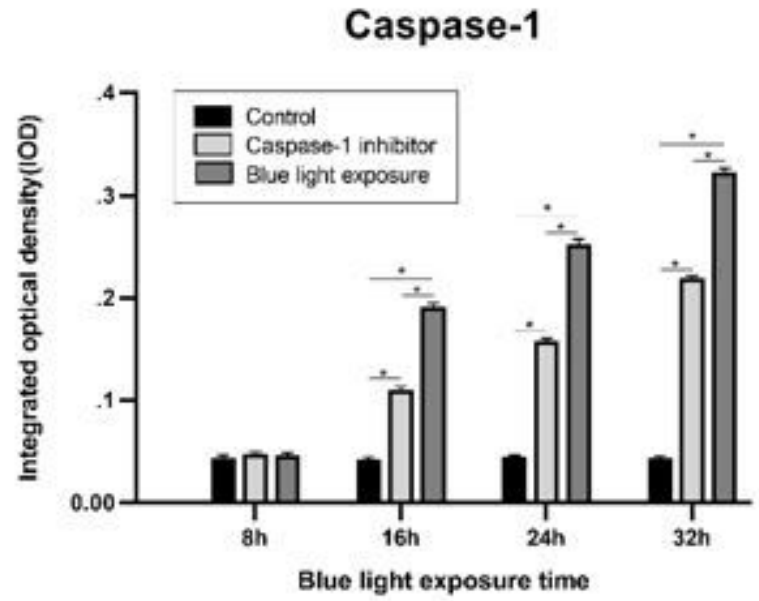

d

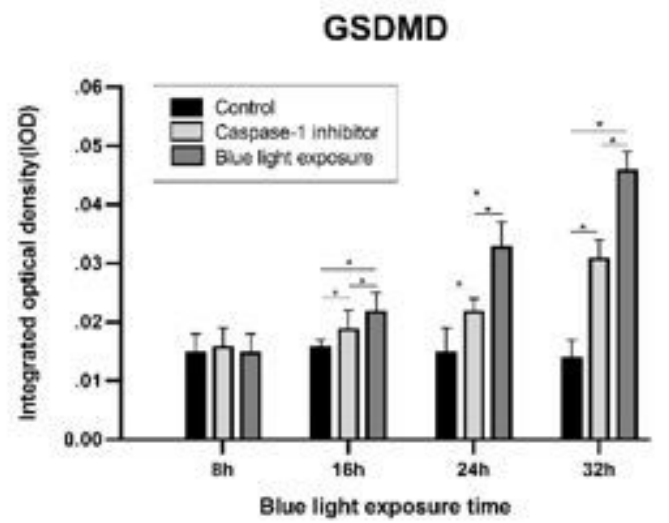

Figure 4

Immunofluorescence analysis of Caspase-1 and GSDMD in HLE-B3 hLECs cells at different time points (a) Representative caspase-1 confocal fluorescence images. FITC-labelled Caspase-1 p20 is green. (b) Integrated optical density (IOD) of caspase-1 p20. (c) Representative GSDMD confocal fluorescence images. (d) IOD of GSDMD. $n=5 .{ }^{*} p<0.05$. 

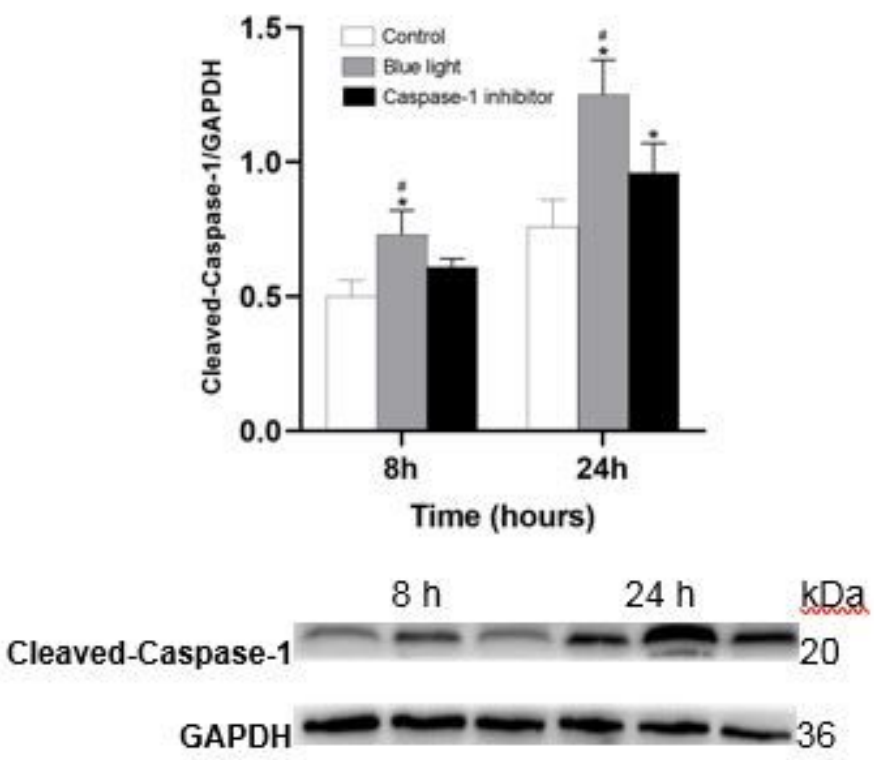

b
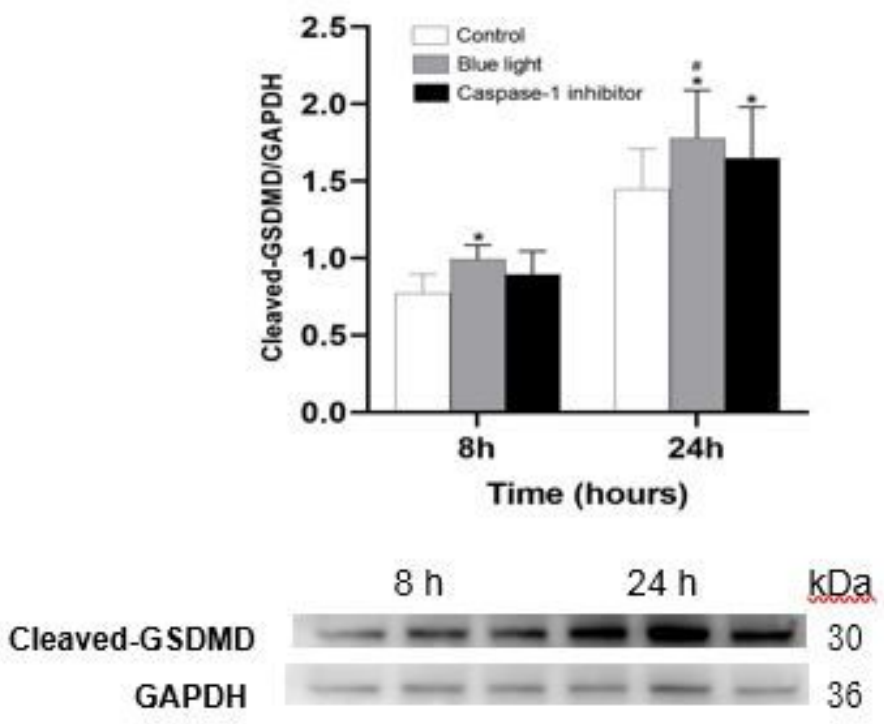

\section{Figure 5}

The protein expression of Caspase-1 and GSDMD in HLE-B3 hLECs using Western blotting (a) The influence of the caspase-1 inhibitor on caspase-1 expression was detected by Western blot. (b) The influence of the caspase-1 inhibitor on GSDMD expression was detected by Western blot. The protein expression levels of caspase- 1 and GSDMD in response to short-wavelength blue light exposure were upregulated at $8 \mathrm{~h}$ and $24 \mathrm{~h}$ compared to those in the control and AC-YVAD-CMK groups $(p<0.05)$, as 
determined by Western blot analysis. However, the expression of caspase-1 and GSDMD in the caspase-1 inhibitor groups decreased in the presence of AC-YVAD-CMK. ${ }^{*} p<0.05$ versus the control group. \#p<0.05 versus the caspase- 1 inhibitor group. The differences were statistically significant.
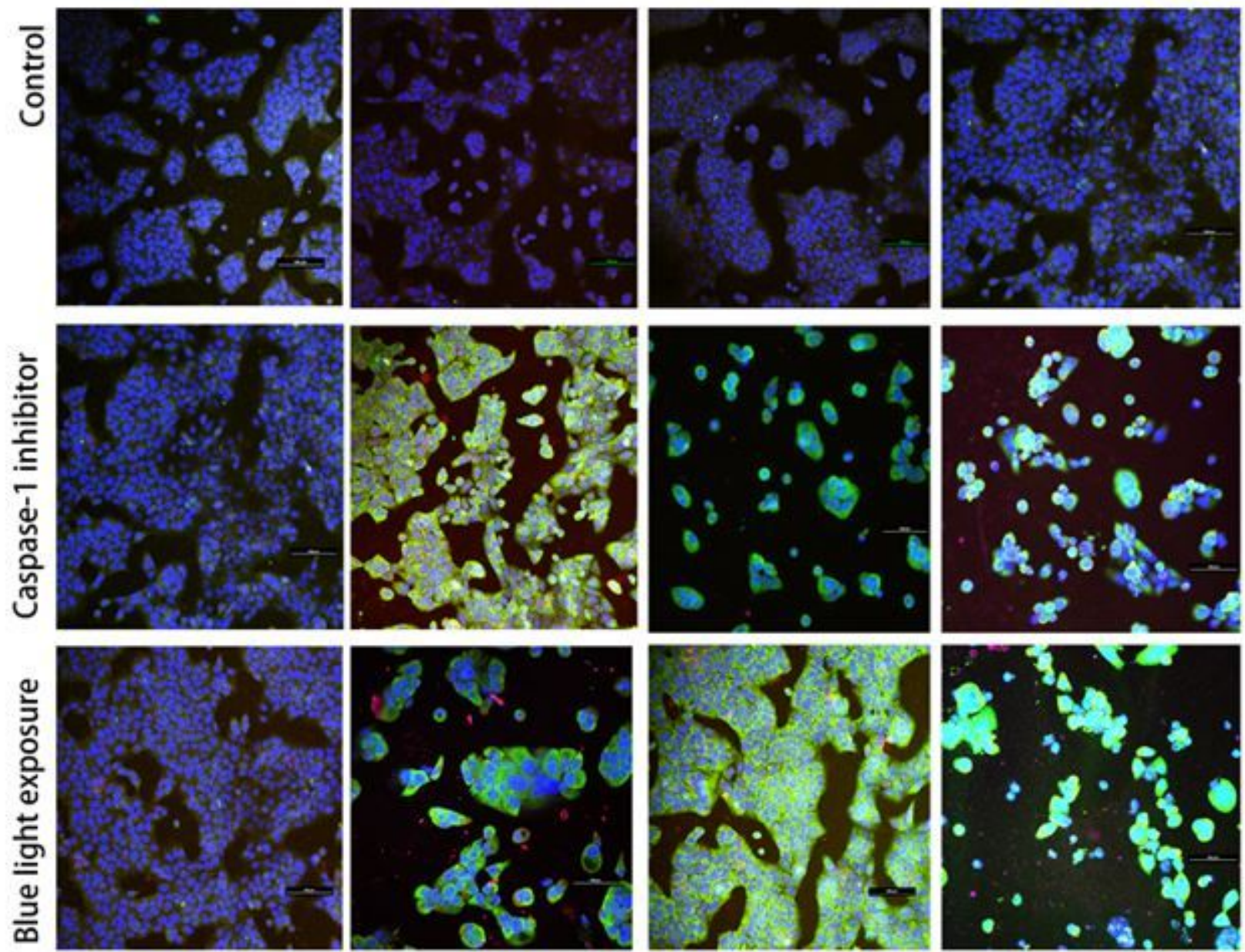

$8 \mathrm{~h}$

$16 \mathrm{~h}$

$24 \mathrm{~h}$

$32 \mathrm{~h}$

Figure 6

Cellular immunofluorescence results of Caspase-1 p20 and GSDMD-N expression in HLE-B3 hLECs at different time points DAPI-stained nuclei are blue, FITC-labelled Caspase-1 p20 is green, and TRITClabelled GSDMD-N is red. Confocal fluorescence images of the control, caspase-1 inhibitor, and shortwavelength blue light groups at different time points. The fluorescence intensity in the control group did not change with time. Both Caspase-1 p20 and GSDMD-N were localized in the cytoplasm. With longer blue light exposure, the expression of Caspase-1 p20 in the cytoplasm gradually increased and localized in a nonspecific position in the cytoplasm. However, the expression of GSDMD-N in the cytoplasm gradually increased, and the localization gradually transferred from the cytoplasm to the cell membrane, but neither protein was expressed in the nucleus. 


\section{Supplementary Files}

This is a list of supplementary files associated with this preprint. Click to download.

- SupplementaryMaterials.docx 